\title{
Experimental Detection of Sign-Reversal Pairing in Iron-Based Superconductors
}

\author{
Jiansheng $\mathrm{Wu}$ and Philip Phillips \\ Department of Physics, University of Illinois at Urbana-Champaign, \\ 1110 W. Green Street, Urbana IL 61801, U.S.A.
}

\begin{abstract}
We propose a modified Josephson corner-junction experiment which can test whether the order parameter in the iron pnictides changes sign between the electron and hole pockets of the Fermi surface.

PACS numbers: $71.10 \mathrm{Hf}, 71.55 .-\mathrm{i}, 75.20 . \mathrm{Hr}, 71.27 .+\mathrm{a}$
\end{abstract}

Iron pnictides represent the newest member of the class of correlated materials in which superconductivity emerges from doping an ordered state $\underline{1,2}$. One of the intriguing proposals for superconductivity in these mutiband systems is that spin fluctuations mediate electron pairing between different regions of the Fermi surface but with different signs for the order parameter ${ }^{3}$. In the unfolded Brillouin zone, the regions of the Fermi surface which are relevant are the electron and hole pockets located at the $M$ and $\Gamma$ points ${ }^{4}$, respectively as illustrated in Fig. (10). The result is a nodeless gap, denoted as $s_{ \pm}$, with a rough momentum dependence of $\cos k_{x} \cos k_{y} \frac{3}{2}$. While the preponderance of the experiments support isotropic nodeless superconductivity $\underline{4,5,6}$ in both the 1111 and 122 materials, the power-law behaviour of the spin-lattice relaxation rate, $T_{1}^{-1} \approx T^{3}$ has been used as a strong indication of line nodes ${ }^{7}$. However, nodeless $s_{ \pm}$pairing has recently been shown to also yield $T^{3}$ behaviour at high temperatures ${ }^{8}$. Fine tuning with disorder is necessary to obtain the $T^{3}$ dependence of $1 / T_{1}$ at low temperatures. Alternatively, superconductivity with multiple gaps can also give rise to such a deviation from the standard BCS exponential fall-off of $T_{1}^{-1}$. In fact, in superconductivity in $\mathrm{Ba}_{0.6} \mathrm{~K}_{0.4} \mathrm{Fe}_{2} \mathrm{As}_{2}$ and $\mathrm{PrFeAsO} \mathrm{O}_{0.89} \mathrm{~F}_{0.11}$ is consistent with at least two gaps with ratios of 2 and 3.2, respectively. Hence, the complete consistency of $s_{ \pm}$pairing with the experimental data is far from settled.

Nonetheless, given the novelty of the $s_{ \pm}$state, it is important to definitively determine its relevance to the pnictides. Although there are some proposals on phase sensitive measurements such as a three layer sandwich structure ${ }^{\frac{9}{}}$, they are not direct probes of the order parameter phase. As phase-sensitive measurements 10 using Josephson interferometry were pivotal in settling the question of the symmetry of the order parameter in the cuprates, we focus here on whether or not such a similar experiment can be performed to falsify the claim that the order parameter in the pnictides has $s_{ \pm}$symmetry. Detecting an $s_{ \pm}$state in the pnictides poses a distinct challenge from discerning the sign change in the $d_{\mathrm{x}^{2}-\mathrm{y}^{2}}$ state in the cuprates because the sign change occurs along the crystal axes 10 .

Central to the design of any standard superconductorinsulator-superconductor (SIS) junction oxide-barrier is the highly directional nature of the transport. Namely a)

b)


FIG. 1: Design of the SQUID junction to test $s_{ \pm}$-wave superconductivity. a) Fermi surface in the folded Brillouin zone to show the principle of design. b) The SQUID design. The left-hand-side of the junction is an iron-based SC which is cut on the [010] (E face), [110] (D face) and [110] (C face) planes. Any two of the planes are connected to a conventional $s$-wave $\mathrm{SC}$ on the right-hand-side through standard oxide-barrier SIS junctions ${ }^{10}$. The magnetic field is perpendicular to the plane.

the junction can only detect the order parameter in the direction perpendicular to the crystal face $\underline{\underline{10}}$. It is for this reason that a standard corner junction can be used to detect the sign change of the $\mathrm{d}_{x^{2}-y^{2}}$ order parameter because the order parameter has a natural alignment along the crystal axes. In this sense, detecting the $\mathrm{s} \pm$ state depicted in Fig. (10) poses a distinctly new challenge because no such alignment of the order parameter and the crystal axes is present. Consider an $s_{ \pm} \mathrm{SC}$ and a conventional $s$-wave SC joined by a weak link. Let $\Delta_{0}$ and $\Delta_{1}$ be the magnitude of the order parameters at the $M$ and $\Gamma$ points, respectively in the iron-based superconductor. The SQUID design is shown in Fig(10). Let us consider the gap on the $M$-pocket which experimentally is less than $\Delta_{1}=25 \mathrm{meV} \stackrel{11}{11}$. A gap of this magnitude corresponds to a wave vector for the centerof-mass of a Cooper pair emanating from the $M$-pocket that is less than $K_{c}=m_{\mathrm{eff}} \Delta_{1} / \hbar p_{F} \approx \frac{0.026}{k_{F}}\left(\frac{m_{\mathrm{eff}}}{m_{e}}\right)\left(\frac{\pi}{a_{0}}\right)^{2}$ where $m_{\mathrm{eff}}, m_{\mathrm{e}}, a_{0} \approx 2.83 \dot{A}, p_{F}$ and $k_{F}$ are the effective mass,electron mass, lattice constant, Fermi momentum and Fermi wave vector respectively. This number is much smaller than the wave vector $Q=(\pi, \pi) / a_{0}$, in the folded Brillouin Zone, by a factor of 3. In this case, regardless of the direction of the Cooper pairs, it is impossible to 
a)

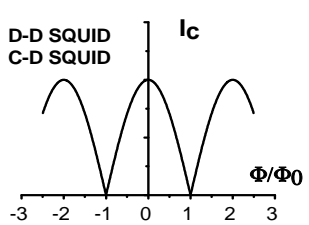

b)

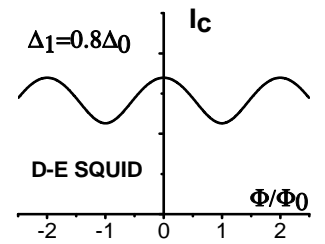

c)

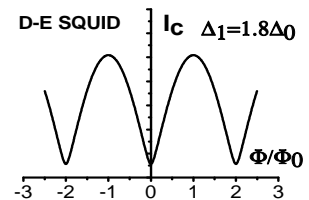

d)

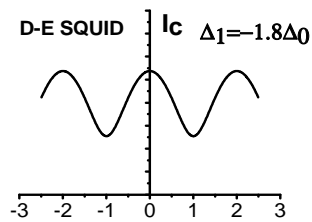

FIG. 2: All possible interference pattern for different $x$ values where $x$ is defined as $\Delta_{1}=-x \Delta_{0}$. a) For junction connecting D-D or C-D faces; b) c) d) are all junction connecting D-E faces. b) $x \in[0,1]$; c) $x \in[1, \infty)$; d) $x \in(-\infty, 0]$.

choose the wave vector $\mathbf{K}_{\mathrm{c}}$ of a Cooper pair emanating from $M$ so that the net wave vector $\mathbf{K}_{\text {net }}$ is perpendicular to the $\mathrm{C}$ or $\mathrm{D}$ faces of the junction in Fig. (10). However, this condition is easily met at the face $\mathrm{E}$ which lies at an angle of $45^{\circ}$ from the horizontal. Such scattering of a Cooper pair from $\Gamma$ to the $M$ point requires Umklapp scattering as the net momentum transfer is $\mathbf{Q}$. By contrast, the order parameter associated with $\Gamma$-pocket in the folded zone can be sensed by all faces. Taking this into account, we compute the associated critical current for a SQUID joining the surfaces C and D, D and D, or D and $\mathrm{E}$. For the former two, the critical current is given by

$2 \Delta_{0} \sin \left(\Phi / \Phi_{0}\right)$ where $\Phi, \Phi_{0}$ is the magnetic flux and flux quantum. This is the standard s-wave result. However, for a D-E SQUID, the situation is different; the critical current,

$$
\sqrt{\Delta_{0}^{2}+\left(\Delta_{0}-\Delta_{1}\right)^{2}+2 \Delta_{0}\left(\Delta_{0}-\Delta_{1}\right) \cos \left(\Phi / \Phi_{0}\right)}
$$

is governed by the magnitude and sign of the order parameter at the $M$ and $\Gamma$ points as depicted in Fig. (2). To recover the standard s-wave result simply requires reversing the sign of $\Delta_{1}$. All the possible interference patterns as a function of $\Phi / \Phi_{0}$ are shown in Fig.(2). For the 1111 pnictide material, there are two hole pockets at the $\Gamma$ point with $\Delta_{0}=(6+12) \mathrm{meV}$ and two electron pockets at $\mathrm{M}$ point with $\Delta_{1}=(12+12) \mathrm{meV}$. This case corresponds to (c) in Fig. 2 $)^{11}$. As all the possible interference patterns differ substantially from the standard s-wave result, this experimental design should offer a definitive test of $s_{ \pm}$pairing in the pnictides.

Acknowledgement J. Wu would like to thank Anthony J. Leggett, Dale J. Van Harlingen, B. Andrei Bernevig and Seungming Hong for their helpful discussions.

Note After this paper was completed, a similar idea was proposed by I. I. Mazin and D. Park in arXiv:0812.4416. We offer here a more complete explanation why along the $D$-face, only holes contribute the current while along the $E$-face, both electrons and holes do. Further, $D-E$ have a $\pi$ phase shift only when the gap on electron Fermi surface is larger than the gap on hole Fermi surface which is not mentioned in arXiv:0812.4416.
1 Cao Wang, Linjun Li, Shun Chi, Zengwei Zhu, Zhi Ren, Yuke Li, Yuetao Wang, Xiao Lin, Yongkang Luo, Shuai Jiang, Xiangfan Xu, Guanghan Cao and Zhu'an Xu, Europhys. Let. 83, 67006 (2008).

2 C. d. l. Cruz, et al., Nature 453, 899 (2008).

3 I. I. Mazin, D. J. Singh, M. D. Johannes, and M. H. Du, Phys. Rev. Lett. 101, 057003 (2008); Kazuhiko Kuroki, Seiichiro Onari, Ryotaro Arita, Hidetomo Usui, Yukio Tanaka, Hiroshi Kontani, Hideo Aoki- Phys. Rev. Lett. 101, 087004 (2008); Kangjun Seo, B. Andrei Bernevig, Jiangping $\mathrm{Hu}$ Phys. Rev. Let. 101, 206404 (2008); Fa Wang, Hui Zhai, Ying Ran, Ashvin Vishwanath, DungHai Lee, arXiv: 0805.3343; D. Parker, O. V. Dolgov, M. M. Korshunov, A. A. Golubov, and I. I. Mazin, Phys. Rev. B 78, 134524 (2008); A. V. Chubukov, D. Efremov, and I. Eremin, Phys. Rev. B 78, 134512 (2008) ; M. M. Korshunov and I. Eremin, Phys. Rev. B 78, 140509(R) (2008); T. A. Maier and D. J. Scalapino, Phys. Rev. B 78, 020514 (2008).

4 Haiyun Liu, Wentao Zhang, Lin Zhao, Xiaowen Jia, Jianqiao Meng, Guodong Liu, Xiaoli Dong, G. F. Chen, J. L. Luo, N. L. Wang, Wei Lu, Guiling Wang, Yong Zhou, Yong Zhu, Xiaoyang Wang, Zhongxian Zhao, Zuyan Xu, Chuangtian Chen, X. J. Zhou, Phys. Rev. B 78, 184514 (2008)
${ }^{5}$ K. Hashimoto, T. Shibauchi, T. Kato, K. Ikada, R. Okazaki, H. Shishido, M. Ishikado, H. Kito, A. Iyo, H. Eisaki, S. Shamoto, Y. Matsuda, Phys. Rev. Lett. 102, 017002 (2009)

${ }^{6}$ Gang Mu, Xiyu Zhu, Lei Fang, Lei Shan, Cong Ren, HaiHu Wen, Chin. Phys. Lett. 25 , 2221 (2008)

7 Yusuke Nakai, Kenji Ishida, Yoichi Kamihara, Masahiro Hirano, Hideo Hosono , J. Phys. Soc. Jpn.77, 073701 (2008)

8 B. Bang and H.-Y. Choi, arXiv: 0808.0302; A. V. Chubukov, D. Efremov, and I. Eremin, Phys. Rev. B 78, 134512 (2008) ; Meera M. Parish, Jiangping Hu, B. Andrei Bernevig, Phys. Rev. B 78, 144514 (2008); Yuki Nagai, Nobuhiko Hayashi, Noriyuki Nakai, Hiroki Nakamura, Masahiko Okumura, Masahiko Machida, New J. Phys. 10 103026(2008)

9 Wei-Feng Tsai, Dao-Xin Yao, B. Andrei Bernevig, JiangPing $\mathrm{Hu}$, arXiv: 0812.0661.

10 D. J. V. Harlingen, Rev. Mod. Phys, 67, 515 (1995)

11 H. Ding, P. Richard, K. Nakayama, K. Sugawara, T. Arakane, Y. Sekiba, A. Takayama, S. Souma, T. Sato, T. Takahashi, Z. Wang, X. Dai, Z. Fang, G. F. Chen, J. L. Luo and N. L. Wang, Europhys. Lett. 83, 47001(2008) 